\title{
Guidewire Length
}

National Cancer Institute

\section{Source}

National Cancer Institute. Guidewire Length. NCI Thesaurus. Code C150371.

The length of the guidewire. 